\title{
Prototyping an S-Band Conformal Line Array Antenna on a Partial Wing Surface
}

This paper was downloaded from TechRxiv (https://www.techrxiv.org).

\section{LICENSE}

CC BY 4.0

SUBMISSION DATE / POSTED DATE

$12-03-2021 / 18-03-2021$

\section{CITATION}

Ford, Alexander (2021): Prototyping an S-Band Conformal Line Array Antenna on a Partial Wing Surface. TechRxiv. Preprint. https://doi.org/10.36227/techrxiv.14210585.v1

DOI 


\title{
Prototyping an S-Band Conformal Line Array Antenna on a Partial Wing Surface
}

\author{
Thomas G. Spence, Alexander L. Ford, Christopher C. Roberts \\ Northrop Grumman Corporation, Baltimore, MD USA \\ \{thomas.spence, alexander.ford, christopher.roberts\}@ngc.com
}

\begin{abstract}
This paper presents the design, prototyping, and testing of an S-Band conformal array on a partial wing surface. The array elements are series fed microstrip patch antennas fabricated entirely through additive manufacturing (AM) technology using a combination of fused deposition modeling and thermal spray. A robust material set of ULTEM 9085 and copper alloy is used for a good balance of mechanical/environmental robustness and RF performance, while also offering a viable path forward for a future fielded design. The focus of this paper is on AM multi-material fabrication, fundamental print settings and material characterization, and antenna testing. AM characterization coupons are utilized to improve the accuracy of the RF antenna model, which showed excellent agreement with the prototype measurements.
\end{abstract}

Keywords-array antenna; conformal antenna; additive manufacturing; patch antenna

\section{INTRODUCTION}

Phased array antennas provide flexible performance not readily achievable through individual antenna elements or fixed beam arrays. The majority of fielded phased arrays possess a planar radiating aperture with a single surface normal vector. Some applications require non-planar or conformal phased arrays to meet system requirements, such as a cylindrical array with a 360 degree field-of-regard [1] or a conformal structurally integrated array on an unmanned aerial vehicle (UAV) [2] to balance aerodynamics and a limited payload volume. The design, analysis, fabrication, and integration of this class of array present a number of challenges not present for planar arrays. In recent years interest is growing in the use of multi-material additive manufacturing (AM) technology to aid in conformal array antenna fabrication and to enable more complex geometry fabrication [2]-[5]. While AM-based fabrication offers a number of compelling benefits it is not without its challenges for a fielding a functional component. AM solutions must be able to meet environmental, structural, thermal, and RF requirements.

The AM technology and materials utilized in [2]-[5] range from a stereolithography photopolymer to a fused deposition modeling ABS material. While they led to very good prototypes, the selected materials are not expected to meet demanding environmental (e.g., high operational temperature) and mechanical (e.g., high tensile strength) requirements of some applications. This paper presents the prototyping of an SBand conformal array that is based on a robust set of AM

(C) 2020 Northrop Grumman. Distribution Statement A: Approved for Public Release; Distribution is Unlimited; \#20-0020; Dated 01/13/20.

978-1-7281-6670-4/20/\$31.00 C2020 IEEE materials and technology. The antenna is formed entirely using AM technology and the materials are specifically selected to offer a viable solution for the stressing objective end-use requirements. A complete AM-based fabrication approach was selected for this prototype for consistency with the objective final implementation. The targeted installation is a structurally integrated portion of a UAV wing or fuselage that has a singly- or doubly-curved outer mold line. The focus of this paper is on the AM multi-material fabrication, fundamental print settings and material characterization, and performance testing.

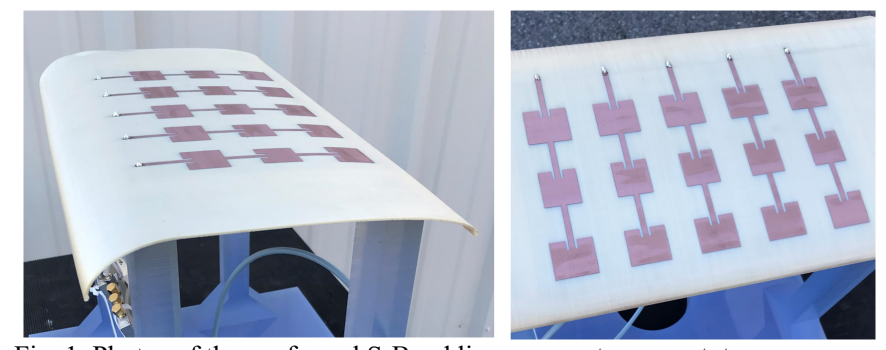

Fig. 1. Photos of the conformal S-Band line array antenna prototype.

\section{ANTENNA DESIGN}

The prototype presented in this paper is a conformal SBand line array that supports beam steering along its length up to 60 degrees. The array elements are series fed microstrip patch antennas that are used to increase the total gain of the array and to narrow the beamwidth along the non-scanned direction [6]. The center-to-center patch spacing along the length and width of the array is $5 \mathrm{~cm}$. The initial series fed patch antenna elements were designed in isolation assuming a flat substrate. The elements were then converted to the conformal array configuration where final spacing and impedance tuning was performed. At the targeted resonant frequency of $3.4 \mathrm{GHz}$ the antenna has a peak realized gain of $15.7 \mathrm{dBi}$ and a total efficiency of $-1.75 \mathrm{~dB}$ (assuming material parameters discussed in Section III). For reference, the efficiency increases by approximately $0.4 \mathrm{~dB}$ if the conductors are replaced by bulk copper in the model.

\section{FABRiCATION \& Process ChARACTERIZATION}

A subscale 5-element line array on a singly-curved substrate was selected for the prototype (Figure 1). Its overall size is $40 \mathrm{~cm} \times 23 \mathrm{~cm} \times 3 \mathrm{~cm}$. Its fabrication is comprised of a 
few basic steps. First, the conformal partial wing surface is fabricated out of $1.3 \mathrm{~mm}$ thick ULTEM 9085 ( $\varepsilon \mathrm{r}=2.8$, $\tan \delta=0.003$ ) using a Statasys Fortus $450 \mathrm{mc}$ [7]. Various print settings and orientations were explored to balance geometry accuracy, surface finish, and repeatability. The selected print orientation for the prototype was to have its long edge perpendicular to the build plate. Next, the ground plane is formed on the underside of the printed part using a copper alloy thermal spray process; the ground plane covers most of the underside and there are small regions void of the copper alloy to accommodate feedthrough of the connector pins. Then the microstrip circuitry is formed on the upper side of the printed part using the same thermal spray process. Printed fiducial targets are used to aid in the circuitry alignment on the two surfaces. Finally, a SMA connector is soldered to each of the array elements. Minimum line widths and gap widths are $3.7 \mathrm{~mm}$ and $2.0 \mathrm{~mm}$, respectively. The conductors have an average thickness of $30 \mathrm{um}$.

A number of process development and characterization coupons were fabricated in parallel with the antenna to guide its fabrication and to improve modeling accuracy. Among them were RF coupons for characterizing the dielectric constant and insertion loss of the selected materials. Several resonator microstrip coupons confirmed the dielectric constant of the printed ULTEM 9085 at $2.8 \pm 0.05$. Differential microstrip line coupons were measured to estimate the insertion loss of copper alloy traces formed on the ULTEM 9085 substrate. Figure 2 shows the measured insertion loss per $\mathrm{cm}$, which takes into the dissipative loss of the substrate, the finite conductivity of the traces and ground plane, and surface roughness. A comparable modeled prediction is overlaid with the measured data. It assumes a finite conductivity of $8.7 \mathrm{e} 6 \mathrm{~S} / \mathrm{m}$ and a smooth surface finish. Additional coupons explored the impact of different surface preparations, circuitry deposition settings, and print orientations. Coupons exposed to thermal cycling (250 cycles; $-40 \mathrm{C}$ to $90 \mathrm{C}$ ) and thermal aging tests $(85 \mathrm{C}$ for 10 days) exhibited excellent stability.

\section{TESTING}

The S-parameters of the fabricated prototype were measured using a vector network analyzer. In Figure 2 very good agreement is observed between the measured and modeled return loss vs frequency. The minimum in the modeled response near $3.4 \mathrm{GHz}$ is the resonant frequency of the antenna. The slight $40 \mathrm{MHz}$ upward frequency shift noted in the measured resonant frequency falls within tolerance range of the measured dielectric constant noted in the previous section.
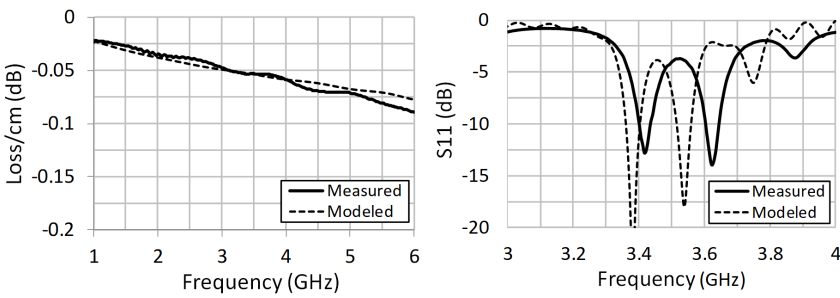

Fig. 2. (Left) Loss per $\mathrm{cm}$ of a $50 \mathrm{Ohm}$ microstrip trace fabricated using the material and processes presented in this paper. (Right) Return loss of the center element of the conformal array prototype.

(C) 2020 Northrop Grumman. Distribution Statement A: Approved for Public Release; Distribution is Unlimited; \#20-0020; Dated 01/13/20.
Individual element and total array radiation patterns were measured in a far-field anechoic chamber. In addition, steered array patterns were generated by analytically phasing the measured individual element data. Realized gain pattern cuts of the array at the resonant frequency are shown in Figure 3. Overall, excellent agreement in the peak gain and sidelobe level is noted compared to the modeled predictions. The peak realized gain of the measured pattern is $0.4 \mathrm{~dB}$ than that of the model; this difference is believed to be attributed to a slightly more focused beam along the E-plane of the array (Figure 3).
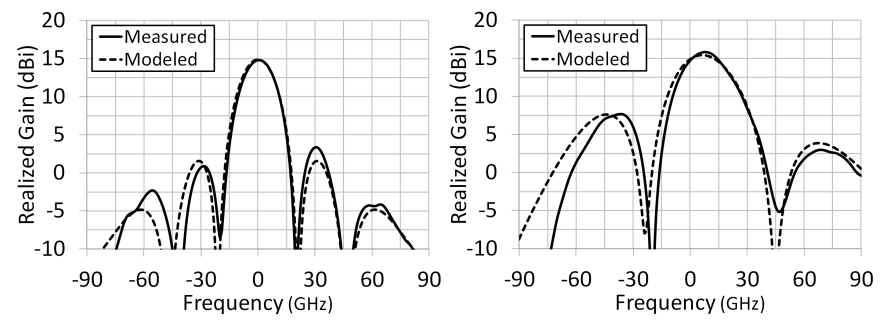

Fig. 3. Realized gain pattern cuts of the 5-element line array prototype at the resonant frequency; (Left) H-plane and (Right) E-plane cuts.

\section{SUMMARY}

This paper presents an overview of the prototyping and testing of a subscale series-fed patch antenna array fabricated entirely using AM technology. The AM technology utilized and the selected material set (ULTEM 9085 and copper alloy) worked well for the antenna prototype and are viable for a future fielded implementation. The measured prototype has demonstrated very good RF performance and its data compare very well with modeled predictions. Additional related followon fully-AM and hybrid-AM array antenna designs are in process.

\section{ACKNOWLEDGMENT}

We would like to thank our colleagues Jacob Darling, Brian Heldmann, Nolan Hoolachan, Chris Huemmer, and Pete Myers for their contribution toward the prototype fabrication and testing.

\section{REFERENCES}

[1] H. Saeidi-Manesh, et al., 'High-Isolation Low Cross-Polarization Phased-Array Antenna for MPAR Application', Radio Sci, vol. 52, 2017.

[2] E. Kreit, et al., 'Printed multilayer conformal x-band antenna array', Flexible and Printed Electronics, vol. 2, 2017.

[3] I. Ehrenberg, et al., "Fabrication of an X-Band conformal antenna array on an additively manufactured substrate", 2015 IEEE Intern. Symp. on Ant. and Prop., pp. 609-610, 2015.

[4] A.J. Fenn, et al., "3D printed conformal array antenna: simulations and measurements", 2016 IEEE Int. Symp. on Phased Array Systems and Technology (PAST), pp. 1-4, 2016.

[5] F. Meng, et al., "3D-printed conformal array patch antenna using a fiveaxes motion printing system and flash light sintering", 3D Printing and Additive Manufacturing, vol. 6, pp. 118-125, 2019.

[6] A. G. Derneryd, 'Linearly polarized microstrip antennas', IEEE Trans. Antennas Propagation, vol. AP-24, pp. 846-851, 1976.

[7] https://www.stratasys.com/ 